\title{
Pane pòsso
}

Ilaria Bonomi

PUBBLICATO: 20 OCTOBER 2020

\section{Quesito:}

Ci è stato chiesto da due lettori, qualche tempo fa, di chiarire l'ambito d'uso e l'eventuale regionalità dell'aggettivo posso, detto di cibi raffermi.

\section{Pane pósso}

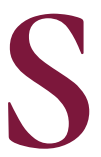

i tratta di una parola dei dialetti dell'area nord-occidentale, non attestata nella tradizione e non registrata dai dizionari della lingua italiana, ma soltanto da quelli dialettali: una parola che però, come vedremo, ha una sua singolare e inattesa presenza nel web.

L'aggettivo è registrato dai dizionari dei dialetti lombardi (póss o altre grafie), piemontesi (pós e altre grafie) e liguri (pöso o pösu o altre grafie) dell'soo e del 'goo con il significato di 'raffermo, non fresco': usato soprattutto in combinazione con pane, ma anche con altri cibi, come le uova, la carne, o anche l'acqua ("Acqua possa, Acqua vecchia", nelle Giunte e correzioni al Vocabolario milanese-italiano di Francesco Cherubini, I843), o addirittura, con uso e significato più ampio di 'passato, avvizzito', riferito a frutta, fiori, abiti che abbiano perduto la loro freschezza (Francesco Angiolini, Vocabolario milanese-italiano, I897).

La voce è documentata ampiamente nei dizionari della Lombardia soprattutto occidentale, compresa la Valtellina e il Canton Ticino, meno nella Lombardia orientale, nei dizionari del Piemonte e della Liguria dal Ponente al Levante.

Se ne trova una precisa definizione in un regolamento della città di Milano del i8ı2, nel quale il pane è posso "quando arriva alle 24 ore dopo la di lui cottura" art. 22 dei "Capitoli per i fabbricatori di pane misto"), e ancora, nel ıوı nel "Giornale della Società italiana d'igiene" (Società italiana di igiene, Associazione fascista per l'igiene Milano) si legge: "Il pane raffermo di 24 ore, il cosi detto pane posso, unisce alla bontà un prezzo più economico, perchè in luogo di 48 cent. al chilo, è venduto a $30-35$ cent.".

La ricerca in Google libri, oltre alle attestazioni appena citate e altre analoghe da testi e documenti soprattutto lombardi dell'8oo e del primo '9oo, offre qualche ricorrenza dell'aggettivo posso in italiano in tempi recenti in romanzi di autori e ambientazione lombardi: per esempio Lucio Mastronardi, Gente di Vigevano (1977), Giovanni Orelli, ticinese, Il treno delle italiane (1995), Maddalena Lissi, TVTB mamma (2007).

Raccolte di filastrocche e la mia memoria di bambina lombarda quando il dialetto si usava ancora documentano pan póss nella conta "Pin pin cavalin / sott' al pè del tavolin / pan pòss pan fresch / indovina che l'è propri quest"; una filastrocca che, se non è più comune tra i bambini milanesi, forse non è del tutto scomparsa, dato che la si trova nelle raccolte di filastrocche, nei siti dedicati a queste e nei siti, abbastanza numerosi, dedicati al dialetto milanese.

Ma il web ci offre una inattesa documentazione di una pur marginale persistenza dell'aggettivo posso, in italiano regionale probabilmente solo settentrionale, anche se una circoscrizione geografica è ardua 
e difficilmente documentabile nel mondo senza confini della rete. Come rilevato, infatti, in una delle domande rivolte al servizio di Consulenza della Crusca (di M. E., che tra l'altro scrive da Londra!) che cita la presenza della voce nel sito di cucina GialloZafferano.it, si trova pan póss nel sito Coquin@ria.it, e biscotti possi qua e là, in alcune ricette (al 20/4/2020, lo trovo nel sito Cottoepostato.it e in un blog).

Infine, due parole sull'origine della parola. L'etimologia accertata anche da recenti studi è dal latino tardo pausare 'cessare, riposare', con un passaggio semantico da 'riposato' a 'raffermo, stantio'; derivazione parallela è quella dello spagnolo pan posado 'pane raffermo', che qualche studioso in passato ha indicato come il punto di origine del lombardo pan poss, vedendolo come uno dei molti ispanismi penetrati nel lombardo durante la dominazione spagnola. E certo la presenza della voce negli altri dialetti nord-occidentali di aree che non hanno avuto la dominazione spagnola nel XVII secolo rafforza l'etimologia diretta dal latino.

\section{Cita come:}

Ilaria Bonomi, Pane pòsso , "Italiano digitale", 2020, XV, 2020/4 (ottobre-dicembre)

DOI: $10.35948 / 2532-9006 / 2020.4411$

\section{Copyright 2020 Accademia della Crusca}

Pubblicato con licenza creative commons CC BY-NC-ND 